\title{
The Possibilities of 5G Communicationusing Xampling Process
}

\begin{abstract}
K.V. Rama Krishna ${ }^{1}$, B. Praneeth Reddy ${ }^{2}$, G. Naresh Kumar ${ }^{3}$
Post Graduate, Department of Electronics and Instrumentation Engineering, Gitam University, Visakhapatnam, India ${ }^{1}$ Assistant Professor, Dept. of Electronics and Instrumentation Engineering, Gitam University, Visakhapatnam, India ${ }^{2,3}$

Abstract: In this paper we are going to explain about the working of $5 \mathrm{G}$ communication and its scope. By using Xampling method we can perform the processing of signals for high frequencies. We are proving it by using Mat-lab Software Tool. We performed the experiments by using the theoretical formulas and implementing them in the MatLab Software GUI Tool. Weare generating the signals by using the formulas and processing them as per the rules of communications systems. By using Mat-Lab software tool we can generate the signals of High frequency $(\mathrm{GHz})$ in the GUI. By using that we can clearly explain the possibilities of implementing the next generation of communication system. By using this paper as reference we can try the further generation of communications also.
\end{abstract}

KeyWords: Xampling, Mat-lab, GUI, High frequency signals, Modulation techniques, Nyquist rate.

1.1 Xampling

\section{INTRODUCTION}

Signal processing methods have changed substantially over the last several decades. The number of operations that are shifted from analog to digital is constantly increasing, leaving the amplifications and fine tunings to the traditional frontend. Sampling theory, the gate to the digital world, is the key enabling this revolution. The process Xampling means we can say it as the advanced version of the sampling theory.

In contrast to the classic subspace sampling, the theory of sampling over UoS (union of subspaces) [4], [5] is still developing. Till now there is no process can reconstruct the signal when it its exact subspace is knows for almost all sampling functions. Here aiming at reducing the sampling rate below Nyquist by exploiting the UoS model.

In this new Xampling method we are using the signals at the rate of Sub-Nyquist [2], [3], which means we are processing the signals below to the sampling rate. This method will be possible by using the sub-Nyquist sampling process, and we can reconstruct that type of signals by using the method of continuous finite block (CTF) [6].

\subsection{Compressing Techniques}

It mainly contains two functions: lowrate analog to digital conversion (X-ADC), lowrate digital to analog conversion (X-ADC) [1]. In both the cases the $\mathbf{X}$ represents the rate of reduction. We can perform the operations of X-ADC by using the random modulator (RD), and modulated wideband converter (MWC) [4] Systems. These methods apply compressed sensing (CR) ideas to reduce the sampling rate of spectrally-sparse signals below the Nyquist rate. The November 2008 special issue of IEEE Signal Processing Magazine reviews existing CR technology. At first sight, the signal models and compression techniques (CR) used seems similar, at least visually.

\subsection{Sub-Nyquist rate}

It may seem that sampling at sampling at Nyquist rate [2], [3]

$\mathrm{f}_{\mathrm{NYQ}}=2 \mathrm{f}_{\max }$

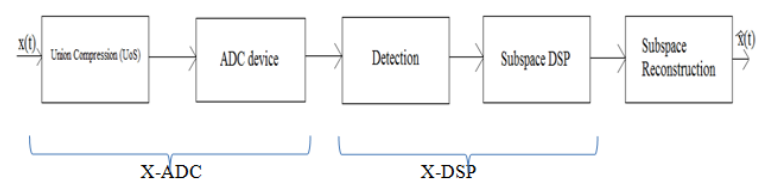

Fig.1: block arrangement of Xampling and its processing

is necessary, since every frequency interval below $f_{\max }$ can potentially contain a transmission of interest. We have the input $\mathrm{x}(\mathrm{t})$. In the above Fig. 1 we can clearly observe the arrangement of the system. On the other side, since each specific $x(t)$ fills only a portion of the Nyquist range [7], one would intuitively expert to be able to reduce the sampling rate below $f_{\mathrm{NYQ}}$. You can observe the block arrangement of Nyquist equivalent of Modulated wideband converter later in Fig 2.2

\section{ADC + DSP + DAC = Min. use of resources}

\section{Modulated Wideband Converter}

In the Fig.2.1 you can observe the arrangement of the modulated wideband converter (MWC) [4]. In this we are using a multiband signal $\mathrm{x}(\mathrm{t})$ has $\mathrm{N}$ frequency bands, with individual widths below $\mathrm{B} \mathrm{Hz}$. The band positions are anywhere below $\mathrm{f}_{\max }$.

The working of modulated wideband converter is initially the input $\mathrm{x}(\mathrm{t})$ passes through frontend of ' $\mathrm{m}$ ' channels. In the $i^{\text {th }}$ channel, $x(t)$ is multiplied by a periodic waveform $\mathrm{p}_{\mathrm{i}}(\mathrm{t})$ with period $\mathrm{T}$, lowpass filtered by $\mathrm{h}(\mathrm{t})$ with cutoff $1 / 2 \mathrm{~T}$, and then sampled at rate $f_{s}=1 / \mathrm{T}$. The basic parameter setting is [4] 
$\mathrm{m} \geq 4 \mathrm{~N}, \quad \mathrm{f}_{\mathrm{s}}=1 / \mathrm{T}, \mathrm{f}_{\mathrm{s}} \geq \mathrm{B}$

Here we require the sampling rate is on the order of $\mathrm{mf}_{\mathrm{s}} \approx 4 \mathrm{NB} \log (\mathrm{M} / 2 \mathrm{~N}+1)$

At first sight, the RD and MWC technologies seem similar, at least in their sampling stages, which involve mixing followed by integration or low-pass filtering as shown in the Fig.2.1

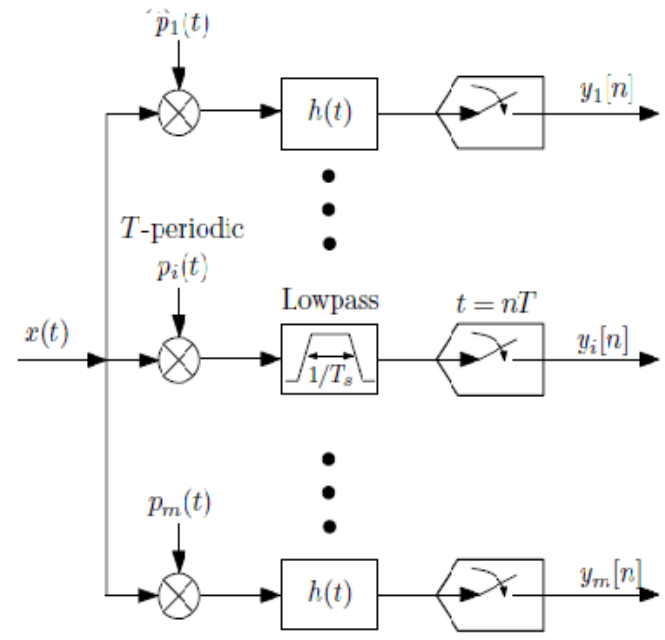

Fig.2.1 Modulated Wideband Converter [4]

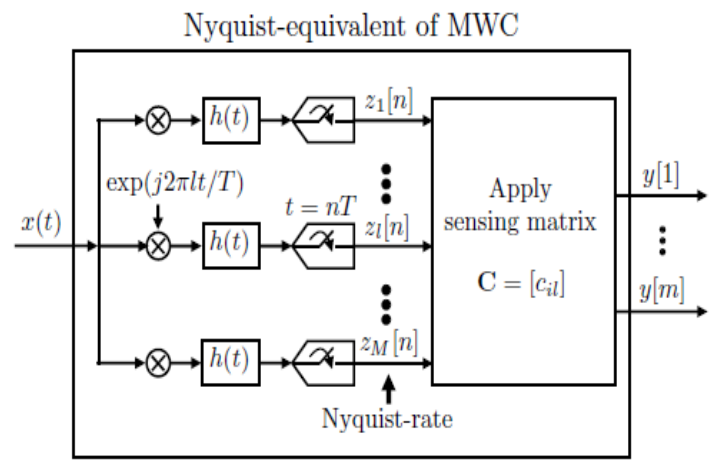

Fig 2.2 Nyquist equivalent of MWC [8]

The software solutions assume that the input is sampled at the Nyquist rate

$$
\mathrm{f}_{\mathrm{NYQ}}=2 \mathrm{f}_{\max }
$$

Which is twice the highest wideband frequency $f_{\max }$. No analog pre-processing is needed and samples can be shared with the subsequent CR stages. Since CR typically operates in a wideband environment, the sampling $f_{N Y Q}$ can be large.

\section{Modulation Techniques}

We have many types of modulation techniques in the digital modulation to process of signals. In the digital modulation we mainly have three types [9]. Those are

- SK (Amplitude Shift Keying)

- SK (Frequency Shift Keying)

- $\quad$ SK (Phase Shift Keying)

In all of them here we are considering four different types. Those are BPSK, QPSK, MSK, MPSK. Because BPSK and QPSK are using methods in the current communication systems. And the other two techniques are better than the older ones for this type of High Frequency signals.

\subsection{Phase Shift Keying}

Phase Shift Keying is a digital modulation scheme that conveys data by changing or modulating the phase of a reference signal. PSK uses a finite number of phases, each assigned a unique pattern of binary digits. Here we are going to use the three sub-methods of the PSK. Those are BPSK, QPSK, MPSK.

\subsubsection{BPSK:-}

It is the simplest form of the Phase Shift Keying. It uses two opposite phases which are separated by the $180^{\circ}$. This method also called as the 2-PSK. Why we use this method? [8],[9], [1].Because this type of modulation is the robust of all PSKs since it takes the highest level of noise or distortion to make the demodulator reach an incorrect decision.

Here we are applying the general form of the BPSK and we are generating the signal and processing the generated signal. The general form of the BPSK is represents by the below equation

$S_{n}(t)=\sqrt{ }\left(2 E_{s} / T_{s}\right) * \operatorname{COS}\left(2 \pi f_{c} t+\pi(1-n)\right) ; n=0,1$.

$\mathrm{S}_{\mathrm{n}}-$ samples; $\mathrm{E}_{\mathrm{s}}-$ energy per symbol; $\mathrm{T}_{\mathrm{s}}-$ symbol duration; $\mathrm{n}=$ number of bits; $\mathrm{f}_{\mathrm{c}}-$ carrier frequency; $\mathrm{t}$ - time period.

We have all the required values in the above equation so by using that input values we can generate the signal and by using the above formula we can modulate the input signal.

\subsubsection{QPSK:-}

It is also called as the quadriphase PSK or 4-PSK. Because it uses the four points which are separated at the angel of $90^{\circ}$. This method can encode two bits per symbol. The mathematical analysis shows in the below equation. It is used to double the data rate compared to the BPSK, while it maintaining the same bandwidth of the signal. The BPSK and QPSK are look like same but the major difference is bit error rate (BER) [11]. In the QPSK the given input signal undergo through the many phase changes. The advantage of QPSK over the BPSK is, QPSK can transmit twice the data rate in given bandwidth compared to BPSK at the same BER. But the communication system engineers feels that the construction of QPSK is complex then the BPSK. But the cost of the QPSK is moderate.

The implementation of QPSK is more simple than the BPSK. The QPSK will implement the higher order of PSK. The general mathematical equation of the QPSK as follows

$\mathrm{S}_{\mathrm{n}}(\mathrm{t})=\sqrt{(}\left(2 \mathrm{E}_{\mathrm{s}} / \mathrm{T}_{\mathrm{s}}\right) * \operatorname{COS}\left(2 \pi \mathrm{f}_{\mathrm{c}} \mathrm{t}+(2 \mathrm{n}-1) \pi / 4\right) ; \mathrm{n}=1,2,3,4$.

Here, by observing the above mathematical equation we can clearly get the $\pi / 4,3 \pi / 4,5 \pi / 4,7 \pi / 4$ [11].

As stated above, here we get the two dimensional signal space with unit basic functions

$\emptyset_{1}(\mathrm{t})=\sqrt{(}\left(2 / \mathrm{T}_{\mathrm{s}}\right) * \cos \left(2 \pi \mathrm{f}_{\mathrm{c}} \mathrm{t}\right) \quad \ldots$ 


$$
\emptyset_{2}(\mathrm{t})=\sqrt{(}\left(2 / \mathrm{T}_{\mathrm{s}}\right) * \operatorname{SIN}\left(2 \pi \mathrm{f}_{\mathrm{c}} \mathrm{t}\right) \ldots
$$

The first function (1) used as the phase component of the signal. The second function (2) used as the quadrature component of the signal. By comparing these basis functions with that for BPSK, shows that QPSK can be viewed as two independent BPSK signals. The main advantage of the QPSK will be, this system can be implemented in a number of ways.

\subsubsection{M-PSK:-}

It is popularly known as the M-ary Phase Shift Keying. This M-ary type of transmission is a type of digital modulation where instead of transmitting one bit at a time, two bits at time or even more bits at a time can transmit simultaneously. This type of transmission results in reduced channel bandwidth.

In this type of modulation sometimes two quadrature carriers are used for modulation. This process is known as the quadrature modulation. Here the output is a baseband representation of the modulated signal. We can apply the scalar or column vector input signals [12]. Base band Mary PSK modulation with a phase shift of ' $\theta$ ' maps an integer ' $n$ ' between 0 and M-1 to the complex value $\exp (\mathrm{j} \theta+\mathrm{j} 2 \pi \mathrm{n} / \mathrm{M})$

The input must be a discrete value.

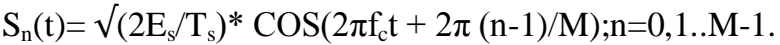

Just like as in the QPSK here also we will have the two functions those are

$$
\begin{aligned}
& \emptyset_{1}(\mathrm{t})=\sqrt{\left(2 / \mathrm{T}_{\mathrm{s}}\right)} * \operatorname{COS}\left(2 \pi \mathrm{f}_{\mathrm{c}} \mathrm{t}\right) \\
& \emptyset_{2}(\mathrm{t})=\sqrt{\left(2 / \mathrm{T}_{\mathrm{s}}\right)} * \operatorname{SIN}\left(2 \pi \mathrm{f}_{\mathrm{c}} \mathrm{t}\right) \quad \ldots
\end{aligned}
$$

The general mathematical formulas of the QPSK and MPSK are look alike, but the major difference will be the phases [13]. Because in the QPSK we are getting the four phases with odd multiples like 1, 3, 5,7. But in this MPSK we will get the random vales as per the input and we have a large limit for the $\mathrm{M}$ in the above equation.

To use the inputs between 0 and M-1 as input values, set the input type parameter to the integer. If the input type parameter is set to Bit and the M-ary number parameter has the form $2^{\mathrm{K}}$ for some positive integers $\mathrm{K}$ then it accepts the binary numbers between 0 and $\mathrm{M}-1$.

\subsection{MSK}

It is the abbreviation for the Minimum Shift Keying. It is a continuous phase frequency keying that was developed in the 1950s. Like as the OQPSK, the MSK is also encoded with alternating bits between quadrature components. The phase of MSK signal is continuous over time and changes $\pi / 2$ during one bit time, where the MSK signal is constant.

The difference between the lower frequency and the higher frequency is almost equal to the half of the bit rate. The maximum frequency deviation is

$$
\delta=0.25 \mathrm{f}_{\mathrm{m}}
$$

$\mathrm{f}_{\mathrm{m}}$ is the maximum modulating frequency. The general mathematical formula for the MSK signal generation is

$S_{n}(t)=\operatorname{COS}\left(2 \pi f_{c} t+b_{k}(t) \pi t / 2 T+\emptyset_{k}\right)$

Sometimes we can write the above equation in the terms of even and odd information. Then the modified formula will be as follows

$S_{n}(t)=a_{I}(t) \operatorname{COS}(\pi t / 2 T) \operatorname{COS}\left(2 \pi f_{c} t\right)-a_{Q}(t) \operatorname{SIN}(\pi t / 2 T)$

$\operatorname{SIN}\left(2 \pi f_{c} t\right)$

$a_{I}(t)$ will be encode even information with duration $2 \mathrm{~T}$.

$\mathrm{a}_{\mathrm{Q}}(\mathrm{t})$ will be encode odd information with duration of $2 \mathrm{~T}$. $b_{k}$ will be +1 for the $a_{I}(t)=a_{Q}(t)$

$b_{k}$ will be -1 for the opposite signs. The $\varnothing_{k}$ will be 0 for $\mathrm{a}_{\mathrm{I}}(\mathrm{t})$ is 1 , and $\pi$ for the remaining cases.

\section{MATLAB}

MATLAB is the short form of the MATrix LABoratory. It is a fourth generation of programming language, developed by math works. Initial version of this software was released in the year of 1984 . This allows matrix manipulations, and plotting the signals and implementing the algorithms, and creating GUI [6]. It can support $\mathrm{C}, \mathrm{C}++$, Java, and python.

\subsection{MATLAB - GUIDE}

This software tool includes GUIDE, which can provide the graphical user interface. We can perform it by using the GUIDE option in the software tool. The GUIDE will provide the point and click controls of the software applications, eliminating the need to learn the programming language or type the commands to run the application.

Actually the GUIDE is simpler when we compared to the coding in the MATLAB. It doesn't require that much of coding techniques. We can directly create the buttons and we can easily add the pictures. We will get an output page for the given designs [14]. The main advantage of this is, by clicking on the output page we will get the required values.

The window which contains the above stated modulation techniques is shown in the Fig.4.1.1, in the picture you can clearly observe the four different modulation techniques we were used and the values what we have applied to generate the signal, like symbol energy and pulse shape and the mathematical formula what we are using there to generate signal.

Here we were provided an option to change the values as per our wish. If you want to apply any values for the symbol energy and pulse shape and SNR and sometimes carrier also, you can apply [6], [15]. As per the given values it will generate the signal by using the mathematical formula.

Otherwise it will take the default values which we have stated. The shape of the signal will be a sine shape or raised cosine shape, because for the high frequency $(\mathrm{GHz})$ signals it will become difficult to understand the data what we are transmitting. 
INTERNATIONAL JOURNAL OFINNOVATIVE RESEARCH IN ELECTRICAL, ELECTRONICS, INSTRUMENTATION AND CONTROL ENGINEERING Vol. 3, Issue 4, April 2015

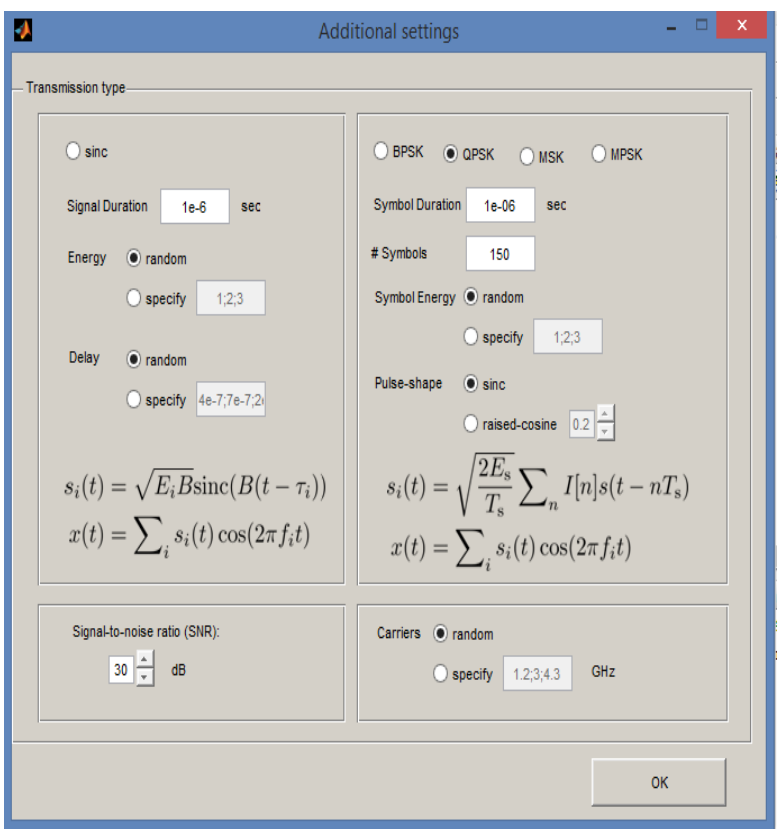

Fig.4.1.1 Modulation techniques window

And the created main window of the entire paper will be shown in the Fig 4.1.2. This mainly having the four subpages. The first stage will be generating the signal by using the applied specifications.

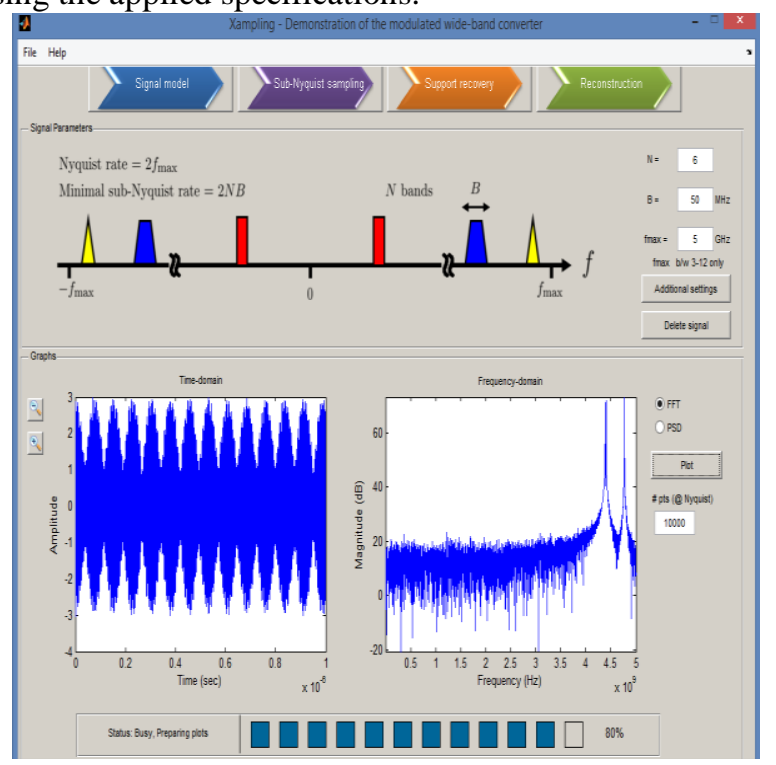

Fig.4.1.2 GUI of the generating signals

Here we can clearly observe the board level design of the input signal using the advanced configuration to treat a multiband model. By clicking on the "additional settings" button we will get the selection of the modulation techniques window, which is shown above in Fig.4.1.1. After selecting the modulation technique now it's time to select the values of $N, B, f_{\max }$. By default, the MATLAB take the given frequency in the Hz's only. But we want $\mathrm{GHz}$ signals as per our requirement [2], [5],[8]. So we will multiply the input value with $10^{9}$ in the background, we should write a simple code for that multiplication.

If we apply the input values $\mathrm{N}=6$ bands and the widths $\mathrm{B}=50 \mathrm{MHz}$, and the $\mathrm{f}_{\max }=5 \mathrm{GHz}$ (should be between 3 to
$15 \mathrm{GHz}$ ), then the spectrum will become $\mathrm{NB}=300 \mathrm{MHz}$. then the sub-nyquist rate will be $2 \mathrm{f}_{\max }=10 \mathrm{GHz}$, and the minimal sub-nyquist rate will be $2 \mathrm{NB}=600$. We will get the sampling rate of $19 \%$ of the nyquist rate [7], [9]. Now click on "plot", then we will get the transfer function of the signal either FFT or PSD as per the applied selection.

The model of the input signal and the transfer function of the signal will be shown in the graphs, which you can see in the above Fig4.1.2. In the graph if you want to change the points in the signal you can change by applying the required value in the place of nyquest points which is below to the plotting of transfer function. After applying the required value at the points we should redraw the signal again. The resolution $\mathrm{f}_{\mathrm{s}}$ can be improved by setting the value $f_{s}<B$ [5], [1]. But this choice is avoided since it increases the computations needed for signal reconstruction when a transmission occupies more than two sequences. The $C R$ settings permit $f_{s}<B$, as only support set $\mathrm{S}$ is needed for sensing the samples.

After generating the signal and its transfer function we will go to the second stage to process the signal. In the second stage the signal should go through the MWC, the working of the MWC is stated above sections. We can observe the working of the MWC through Fig.2.1. The screenshot of the second stage is shown in the below figure 4.1.3. At the right side top we have $\mathrm{m}$ and $\mathrm{M}$ values, and just below to that we get the cut-off frequency and sampling rate and total sampling rate and nyquist rate of the applied signal.

If you want to change the number of samples we can change it. After applying all the values click "generate samples". Then in the background the samples will get generated, we can't see the processing and result samples. But the sample values and its respective calculations will be done without any errors.

During the third stage we are going to do the recovery process. In this step we are using the continuous to finite block (CTF) to recover the samples [4], [12], [13], [15]. We can consider this as the receiver for the system. Here we already have the original samples and after running this third stage we will get the recovered samples of the signal. And we will compare those two original and the recovered values and if they are matched then the operation will be success, if not it shows that the recovery got failed, which will happen in very rare cases if we did any mistakes.

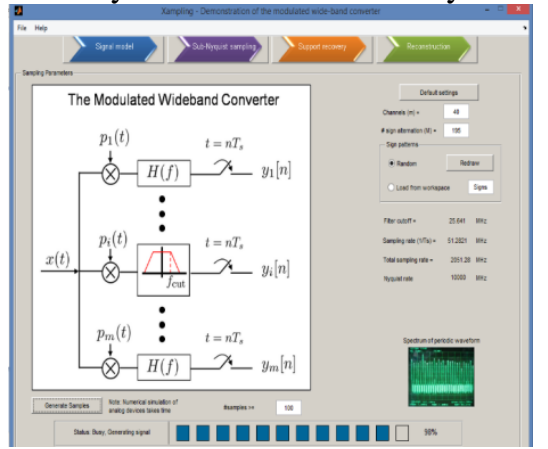

Fig.4.1.3. MWC processing stage window 
The fourth and last stage will be to reconstruct the input a simple and fixed analog frontend. Besides sensing, the signal after receiving the signal. In this stage we are using system also provides CR reception path [10], [11]. Our the pseudo inverse method to reconstruct the samples of design is efficiently realized in hardware and introduces the signal. Here we are applying the input samples at the only light computational loads.

rate of $1 / \mathrm{T}_{\mathrm{s}}$ [3], [4], [14]. The output signals will be having $2 \mathrm{~N}$ baseband sequences. Here we get the output samples at the rate of nyquist rate of the input signal $x(t)$. After that we will get the output of the reconstructed signal from input. Now we can compare the two signals, input and reconstructed signal.

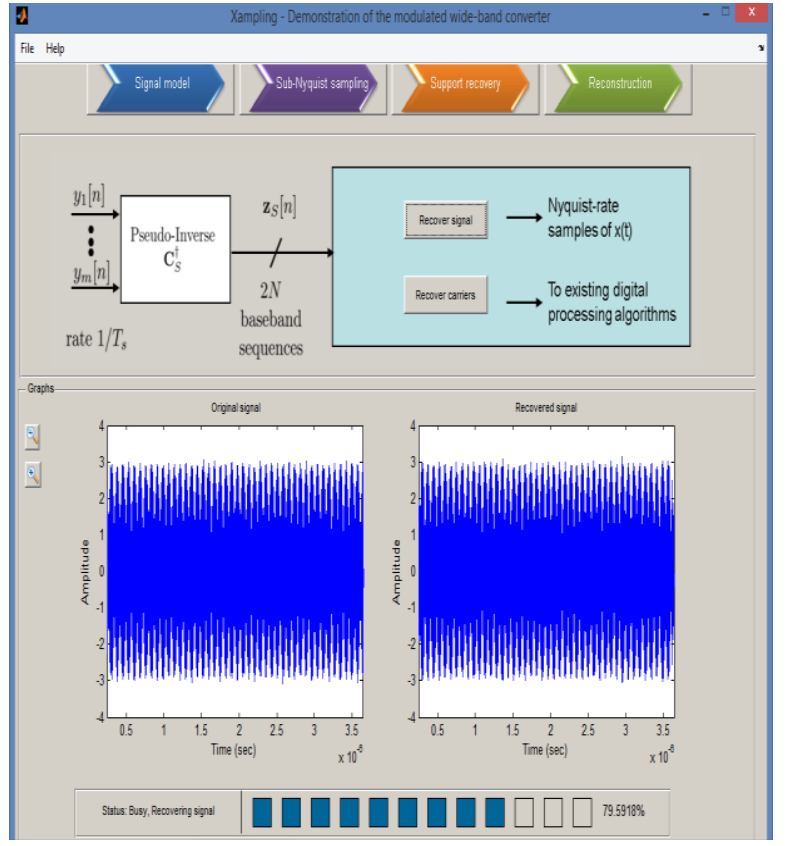

Fig.4.1.4 process of reconstruction window

We can observe the similarities and differences in those two signals. In the above Fig.4.1.4. you can observe the screenshot of the reconstruction. We have proven that my proposed system will suitable to transmit and reconstruct the high frequency signals.

\section{CONCLUSION}

We are proposing this system after testing many types of input values. In the first stage of the GUI [5], in Fig.4.1.2. right side top we are stated the condition for the range of input as 3-15 GHz. The applied frequency does not exceed the maximum value. If we apply the input value below to the given condition of range then also the system will work, but those limits of frequencies are already using in the present communication systems [4], [13], [15]. If the applied frequency is exceeded than the maximum frequency, for the applied modulation techniques it will become very difficult to process them. Because of that we will get the errors all the time and the reconstruction is almost not possible.

This paper proved that the possibilities of the next generation of the communication. We have demonstrated a lowrate, efficient spectrum sensing mechanism that can reliably determine inactive bands over a wide span of the spectrum with respect to the existing sensing strategies, our approach processes a mixedanalog-digital design, with
Hardware experiments report fast and accurate spectrum sensing due to the low sampling rate. Future work should address various hardware related aspects, including how to design the chips, so it can later be embedded into existing mobile platforms.

\section{REFERENCES}

Our sincere thanks to Moshe Mishali and Y.C.Eldar, for their helping and motivating IEEE paper on Xampling.

[1] Y.C. Eldar and Mishali, "Beyond bandlimited Sampl-ing", IEEE Signal process. Mag., vol.26, no.3, May 2009.

[2] C.E. Shannon, "Communication in the process of noise", proc. IRE, vol. 37, Jan 1949.

[3] H. Nyquist, "Certain topics in telegraph transmission theory", Trans. AIEE, vol.47, Apr 1928.

[4] Y.C. Eldar and Mishali, "From theory to practice: sub-nyquist sampling of sparse wideband analog signals", IEEE J.sel. Topics sgnal process, vol. 4, no. 2, Apr 2010.

[5] Y.C. Eldar and Mishali, "Robust recovery of signals from a structured union of subspaces", IEEE Trans. Inf. Theory, vol. 55, no. $11 \mathrm{pp}$, Nov 2009.

[6] Scott T. Smith, "Matlab Advanced GUI Development”, Jun 2006.

[7] I.Budiarjo, H. Nikookar and L.P.Ligthart, "Cognitive radio modulation techniques”, IEEE signal process. Mag., Vol 25, no. 6, 2008.

[8] D.D Ariananda, M.K.Lakshmanan and H.Nikoo, "A survey on spectrum sensing techniques for cognitive radio", in proc. $2^{\text {nd }}$ Int. Workshop Cognitive Radio and advanced spectrum management, May 2009.

[9] T.Yucek and H.Arslan, "A survey of spectrum sensing algorithms for cognitive radio applications", IEEE Commu. Surveys Tutorials, vol. 11, pages 116-130, 2009.

[10] M.Mishali[online],http://www.techion.ac.in/ moshiko/hardware.ht $\mathrm{ml}$

[11] Y.C.Eldar[online], http://webee.technion.ac.in.il/ Sites/People/ YoninaEldar/ Hardware.html

[12] I.F.Gorodnitsky and B.D.Rao, "Sparse signal reconstruction from limited data using FOCUSS: A re-weighted minimum norm algorithm”, IEEE Trans. Signal Process, vol. 45, no. 3, Mar 1997.

[13] M.Mishili, Y.C.Eldar, and A.Elron, "Xampling: Signal acquisition and processing in uniform subspaces", CCIT Report no. 747, Oct 2009.

[14] A.Petraglia and S.K.Mitra, "High speed A/D conver-sion incorporating a QMF bank”, IEEE Trans. Instrum, vol. 41, Jun 1992.

[15] Z.Yu, X.Chen, S.Hoyos, B.M.Sadler, J.Gong, and C.Qian, "Mixed signal parallel compressive spectrum sensing for cognitive radios", International Journal of Digital Multimedia Broadcasting, 2010. 